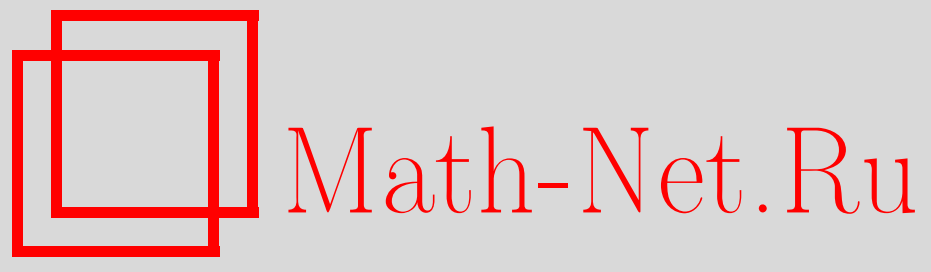

В. М. Нежинский, Многомерный аналог теории Гусарова одномерных узлов, УМH, 2008, том 63, выпуск 1, 165166

DOI: https://doi.org/10.4213/rm9123

Использование Общероссийского математического портала Math-Net.Ru подразумевает, что вы прочитали и согласны с пользовательским соглашением http://www .mathnet.ru/rus/agreement

Параметры загрузки:

IP : 54.205 .225 .156

26 апреля 2023 г., 15:29:18

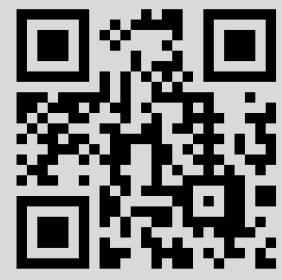




\section{Многомерный аналог теории Гусарова одномерных узлов}

\section{В. М. Нежинский}

Для сферических узлов коразмерности большей двух определены и, насколько это оказалось возможным, изучены аналоги инвариантов конечной степени и групп Гусарова одномерных узлов.

Мы будем считать фиксированными натуральные числа $n$ и $p$ такие, что $n-p>2$.

1. Группа $C(p, n)$ и ее фильтрация. Пусть $r$ - натуральное число. Защеплением типа $(r, p, n)$ называется последовательность гладких вложений $\left(f_{1}: S^{p} \rightarrow S^{n}\right.$, $\left.f_{2}: S^{p} \rightarrow S^{n}, \ldots, f_{r}: S^{p} \rightarrow S^{n}\right)$ с попарно непересекающимися образами; зацепление типа $(1, p, n)$ называется также узлом типа $(p, n)$. Зацепление $\left(f_{1}, f_{2}, \ldots, f_{r}\right)$ называется брунновым, если любое его подзацепление $\left(f_{1}, \ldots, f_{i-1}, f_{i+1}, \ldots, f_{r}\right)$ изотопно стандартному тривиальному зацеплению (для $1 \leqslant i \leqslant r$ ). Хорошо известно (ср., например, [1; п. 9.3]), что множество изотопических классов брунновых зачеплений типа $(r, p, n)$ образует абелеву группу относительно покомпонентного связного суммирования. Эту группу мы будем обозначать через $C(r, p, n)$, а если $r=1$, то и через $C(p, n)$.

Определим гомоморфизм $f_{r}(p, n): C(r, p, n) \rightarrow C(p, n)$ правилом, сопоставляющим классу любого зацепления типа $(r, p, n)$ класс (какого-нибудь) узла типа $(p, n)$, получаемого из этого зацепления (последовательным) связным суммированием всех его компонент. Мы полагаем $F_{r}(p, n)=f_{r}(p, n)(C(r, p, n))$. Заметим, что, поскольку отображение $f_{1}(p, n)$ тождественно, $F_{1}(p, n)=C(p, n)$.

Для любого натурального числа $k$ положим $d(k)=(n-p-2) k+1$. Далее, для любой последовательности целых неотрицательных чисел $k_{1}, \ldots, k_{r}$ таких, что $k_{1}+$ $\cdots+k_{r} \geqslant 1$, положим

$$
e\left(k_{1}, \ldots, k_{r}\right)=\frac{1}{k_{1}+\cdots+k_{r}} \sum_{d \mid k_{i}} \mu(d)\left[\frac{\left(\left(k_{1}+\cdots+k_{r}\right) / d\right) !}{\left(k_{1} / d\right) ! \cdots\left(k_{r} / d\right) !}\right],
$$

где $\mu$ - функция Мёбиуса (суммирование производится по всевозможным общим делителям $d$ чисел $\left.k_{1}, \ldots, k_{r}\right)$. Наконец, положим $\widetilde{\pi}(r, p, n)=\bigoplus\left[\pi_{p}\left(S^{d\left(k_{1}+\cdots+k_{r}\right)}\right) \oplus\right.$ $\left.\cdots \oplus \pi_{p}\left(S^{d\left(k_{1}+\cdots+k_{r}\right)}\right)\right]$, где число слагаемых в квадратных скобках равно $e\left(k_{1}, \ldots, k_{r}\right)$, а внешнее суммирование производится по всевозможным последовательностям целых чисел $k_{1}, \ldots, k_{r}$ таким, что (для некоторого натурального числа $s \leqslant r$ ) $k_{s}=0, k_{i} \geqslant 1$ при $i<s$ и $k_{i}>1$ при $i>s$.

ОСНовная теОРема. Пусть $n u p$ - натуралъные числа такие, что $n-p>2$, $u r$ - еще одно натуралъное число. Тогда: 1) $F_{1}(p, n) \supset F_{2}(p, n) \supset F_{3}(p, n) \supset \cdots$; 2) $F_{1}(p, n)=C(p, n)$; 3) $F_{2}(p, n) \subset$ Ker $E$, и если $4 p<3 n-6$, mo $F_{2}(p, n)=$ Ker $E$, где $E: C(p, n) \rightarrow C(p, n+1)$ - гомоморфизм надстройки (определение надстройки имеется, например, в $[2$; п. 6.1]); 4) если $r>p /(2(n-p-2))+1$, то существует канонический эпиморфизм $\tilde{\pi}(r, p, n) \rightarrow F_{r}(p, n), u$, следовательно, если $r>(p-1) /(n-$ $p-2)+1$, то группа $F_{r}(p, n)$ тривиальна.

2. Аналоги групп Гусарова. Для любого натурального числа $r$ мы полагаем: $G_{r}(p, n)=C(p, n) / F_{r}(p, n)$. Что эти группы - аналоги групп Гусарова, прямо следует из сравнения нашего определения с определениями соответствующих групп одномерных узлов, содержащимися в [3] и [4]. Заметим (в этом состояла основная трудность), что удовлетворительного аналога определения групп Гусарова, содержащегося в его пионерской работе [5], построить не удается: в его определениях принципиально важно, что узлы одномерны.

Из нашей основной теоремы следует, что $G_{1}(p, n)=0$, что если $4 p<3 n-6$, то $G_{2}(p, n)=C(p, n) / \operatorname{Ker} E$, что если $r>(p-1) /(n-p-2)+1, m_{0} G_{r}(p, n)=C(p, n)$, и, 
наконец, что существуют канонические эпиморфизмы $\cdots \rightarrow G_{3}(p, n) \rightarrow G_{2}(p, n) \rightarrow$ $G_{1}(p, n)$. Заметим, что последнее утверждение является прямым аналогом первой части теоремы 3.3 статьи [5] и что предпоследнее утверждение является новым, т. е. подобное равенство в теории одномерных узлов отсутствует.

Пример. Для любого натурального числа $k$ группа $G_{r}(4 k-1,6 k)$ тривиальна при $1 \leqslant r \leqslant 3$ и изоморфна $\mathbb{Z}$ при $r \geqslant 4$. Это - прямое следствие результатов, содержащихся в [1], [2].

3. Аналоги инвариантов конечной степени. Пусть $A$ - коммутативная группа. Назовем (аддитивным изотопическим) инвариантом узлов типа $(p, n)$ со значениями в группе $A$ гомоморфизм $\varphi: C(p, n) \rightarrow A$. Пусть, далее, $r$ - натуральное число; мы будем говорить, что инвариант $\varphi$ имеет степень, не превосходящую числа $r$, если $F_{r}(p, n) \subset \operatorname{Ker} \varphi$. В основе этого определения лежит фундаментальное свойство инвариантов конечной степени, см. [5; лемма 5.1]. (Заметим, что наш инвариант степени, не превосходящей числа $r$, - это аналог инварианта Гусарова из [6] степени, не превосходящей числа $r-2$.) Независимое прямое определение, аналогичное определению из [6], построить не удается: в упомянутом определении существенно, что узлы одномерны (ср. соответствующее замечание п. 2 настоящей заметки).

Обозначим (для любого натурального числа $i$ ) через $G_{i}$ пространство непрерывных отображений $S^{i-1} \rightarrow S^{i-1}$ степени 1 , через $G$ - индуктивный предел последовательности $G_{1} \hookrightarrow G_{2} \hookrightarrow G_{3} \hookrightarrow \cdots$, через $S O$ индуктивный предел последовательности $S_{1} \hookrightarrow \mathrm{SO}_{2} \hookrightarrow \mathrm{SO}_{3} \hookrightarrow \cdots$ и положим $H(p, n)=\pi_{p}\left(G ; S O, G_{n-p}\right)$. Хорошо известно (см. [1], [2] и [7]), что: существует канонический изоморфизм $\psi: C(p, n) \rightarrow H(p, n)$; для $r>1$ существуют коммутативная (гомотопическая) группа $H(r, p, n)$ и изоморфизм $\psi: C(r, p, n) \rightarrow H(r, p, n)$.

Определим гомоморфизм $f_{r}^{\prime}(p, n): H(r, p, n) \rightarrow H(p, n)$ формулой $f_{r}^{\prime}(p, n)=\psi \circ$ $f_{r}(p, n) \circ \psi^{-1}$ и положим $A(r, p, n)=H(p, n) / f_{r}^{\prime}(p, n)(H(r, p, n))$. Определим изотопический инвариант узлов типа $(p, n)$ со значениями в группе $A(r, p, n)$ как композицию гомоморфизма $\psi$ и проекции $H(p, n) \rightarrow A(r, p, n)$. Ясно, что этот инвариант является (универсальным) инвариантом степени, не превосходящей числа $r$. Это многомерный аналог части а) теоремы 2.2.6 работы [8] и последнего замечания шестого абзаца п. 0.4 той же работы.

Автор благодарит С. С. Подкорытова за полезные советы.

\section{Список литературы}

[1] A. Haefliger, Comment. Math. Helv., 41:1 (1966), 51-72. [2] A. Haefliger, Ann. of Math. (2), 83:3 (1966), 402-436. [3] T. B. Stanford, J. Knot Theory Ramifications, 9:2 (2000), 213-219; arXiv: math/9807161. [4] A. Yasuhara, Fund. Math., 190 (2006), 289-297; arXiv: math/0412489v1. [5] М. Н. Гусаров, Записки науч. сем. ПОМИ, 208 (1993), 152-173; англ. пер.: M. N. Gusarov, J. Math. Sci., 81:2 (1996), 2549-2561. [6] М. Н. Гусаров, Записки науч. сем. ПОМИ, 193 (1991), 4-9. [7] В. М. Нежинский, Изв. АН СССР. Сер. матем., 48:1 (1984), 127-154; англ. пер.: V. M. Nezhinskii, Math. USSR-Izv., 24:1 (1985), 121-150. [8] М. Н. Гусаров, Инварианты конечной степени и п-эквивалентность зачеплений, Дис. ... канд. физ.-матем. наук, Санкт-Петербург, 1997.

В. M. Нежинский (V. M. Nezhinskii)

Санкт-Петербургский государственный университет E-mail: nezhin@pdmi.ras.ru
Представлено И. А. Дынниковым Принято редколлегией 19.12.2007 